\title{
Verfilmung und Digitalisierung der Sammlung Ryhiner
}

\author{
Thomas Klöti
}

\section{Die Sammlung}

Der Berner Staatsmann und Geograph Johann Friedrich von Ryhiner (1732-1803) stellte um 1800 eine weltweite, nach wissenschaftlichen Kriterien aufgebaute geographische Sammlung zusammen, die zirka 16.000 Landkarten, Pläne und topographische Ansichten aus dem 16. bis frühen 19. Jahrhundert umfasst. Die Sammlung gelangte 1867 als Geschenk an die damalige Stadtbibliothek Bern und zählt heute zu den wertvollsten, privat angelegten Kartensammlungen des 18. Jahrhunderts.

\section{Das Erschließungsprojekt}

Zwischen 1994 und 1998 wurden die Bestände der Sammlung in einem viereinhalbjährigen Unternehmen erschlossen (http://www.stub.unibe.ch/stub/ryhiner). Das Projekt beinhaltete insbesondere bestandeserhaltende Massnahmen (Restaurierung, farbige Mikroverfilmung) sowie den Aufbau einer allgemein zugänglichen Datenbank. Die Inventarisierungsarbeit schloss an vergleichbare karto-bibliographische Projekte an, die in Deutschland gleichzeitig durchgeführt wurden. Aufgrund dieser bibliothekarischen, restauratorischen und wissenschaftlichen Erschließungsarbeiten ist der Forschung eine reichhaltige Quelle zugänglich gemacht worden.

\section{Nutzung und Bestandessicherung}

Mit dem Nachweis der Bestände in einer öffentlich zugänglichen Datenbank sowie der Präsentation der Sammlung im World Wide Web gingen wir davon aus, dass mit einer verstärkten Nutzung der Sammlung zu rechnen sei. Ein besonderes Gewicht wurde daher auf das Benutzungs- sowie auf das Konservierungs- bzw. Restaurierungskonzept gelegt. Mit der farbigen Mikroverfilmung verfolgten wir zwei Ziele: Im Hinblick auf den Kulturgüterschutz wurde eine Sicherheitskopie verfertigt, die im Kulturgüterschutzraum des Staatsarchivs des Kantons Bern aufbewahrt wird. Diese Kopie wurde vom Bundesamt für Zivilschutz subventioniert, da es sich um eine Maßnahme im Sinne des Kulturgüterschutzes handelt. Eine zweite Kopie dient als Arbeitskopie, die wir dem Benutzer anstelle der Originalkarten, Plänen und Ansichten zur Verfügung stellen. Für die Benutzung der Mikrofilme stellen wir einen Reader/Printer für schwarzweiss Kopien in den Formaten A4 und A3 sowie ein wei- 
teres Mikrofilmlesegerät, die jeweils über eine Mattscheibe in der Grösse von A2 quer verfügen, bereit.

Bei der Suche nach einem geeigneten Mikrofilmverfahren liessen wir uns vom Staatsarchiv Bern beraten. Wir entschieden uns für das Filmmaterial IlfochromeMicrographic. Das gewählte Verfahren ist alterungsbeständig und erzielt eine Farbwiedergabe in höchster Qualität, wobei der Mikrofilm über einen enormen Informationsspeicher verfügt. Das Auflösungsvermögen des Films beträgt über 300 Linienpaare pro Millimeter (lp/mm), wobei in der Praxis, bedingt durch die Optik, 140 180 lp/mm erzielt werden. Der farbige Mikrofilm kann auch - als Zwischenoriginal - gescannt werden, womit durch dieses "hybride" Verfahren auch sämtliche Möglichkeiten der Weiterverwendung von digitalen Bildern offen stehen. Diese digitalen Bilder können dann zum Beispiel auf CDs gespeichert oder auf einem Server der Öffentlichkeit zugänglich gemacht werden.

Mit der Durchführung der Arbeiten war die Firma Gubler in Märstetten betraut, die auf dem Gebiet der Kulturgüterverfilmung eine grosse Erfahrung aufweist. Das Verfilmen erfolgte auf Kleinbild Rollfilmen, die anschließend in Jackets abgepackt wurden. Beim Verfilmen wurden ein Maßstab, ein Farb- und Graukeil sowie die Signatur mitbelichtet. Für den erforderlichen Transport der Bände liessen wir ein Spezialbehältnis aus Hartholz anfertigen, das stossfest, wasserdicht und feuerhemmend wirkt. Für den Transport von Kulturgütern ist eine derartige Schutzmaßnahme unabdingbar.

\section{Digitalisierung}

Bei der Budgetierung des Vorhabens gab es noch keine geeignete Lösung für die Digitalisierung von 16.000 Bildern im Bibliotheksbereich. Da auf diesem Gebiet eine rasante Entwicklung zu beobachten war und weiterhin zu beobachten ist, gingen wir von der Voraussetzung aus, dass eine Digitalisierung bereits während oder zumindest im Anschluss an das laufende Projekt aktuell werden könnte.

In einem ersten Schritt machten wir uns im Jahre 1994 mit den neuen Möglichkeiten vertraut. Anlässlich der Ausstellung "Raum in der Zeit" zeigten wir in einer Vitrine den Sammelatlas "Katalonien". Die dazugehörigen Mikrofilme projizierten wir mit einem Diaprojektor an die Wand. Dieselben Mikrofilme dienten zudem als Vorlage für eine Foto-CD, die gleichzeitig auf einem Monitor abgespielt wurde.

An der 9. Konferenz der Groupe des Cartothécaires de LIBER, die vom 26.30.9.1994 an der ETH-Zürich in Zürich und Glarus zum Thema "Kartenbibliothekswesen im Wandel" durchgeführt wurde, konnte mit dem Referat von Martin Gubler und Thomas Klöti "Der farbige Mikrofilm: Zwischenoriginal für konventionelle und digitale Bildarchivierungssysteme" das Vorhaben erstmals der bibliothekarischen Fachwelt präsentiert werden. In der Poster-Session wurde auch die Foto-CD mit den Bildern des Sammelatlas "Katalonien" gezeigt. 
Ein weiterer Schritt erfolgte am 2. Februar 1995. Dank des Einsatzes und der Fachkenntnisse der Projektmitarbeiterin Caroline Hablützel realisierten wir einen Webauftritt für die Sammlung Ryhiner. Der virtuelle Zugang im World Wide Web (http://www.stub.unibe.ch/stub/ryhiner/ry-dtl.html) erlaubte uns, die Sammlung sowie das Forschungsprojekt weltweit vorzustellen (siehe Abb. 1), wobei der Zugriff auf das Kernstück der Arbeit, die damals im Aufbau begriffene Datenbank, ermöglicht wurde.

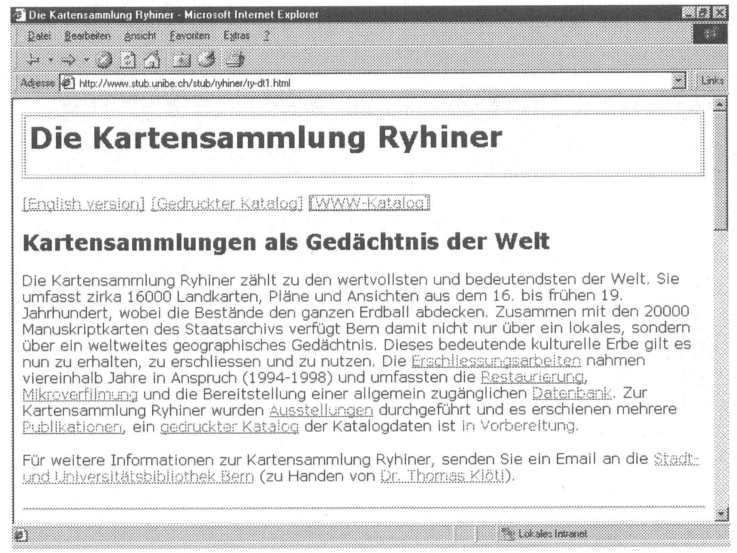

Abb. 1: Homepage der Kartensammlung Ryhiner

Für die textfreie Suche hinterlegten wir der verweis-sensitiven Graphik „Die Schweiz und ihre Kantone" Links auf die entsprechenden HTML-Seiten (Abb. 2).

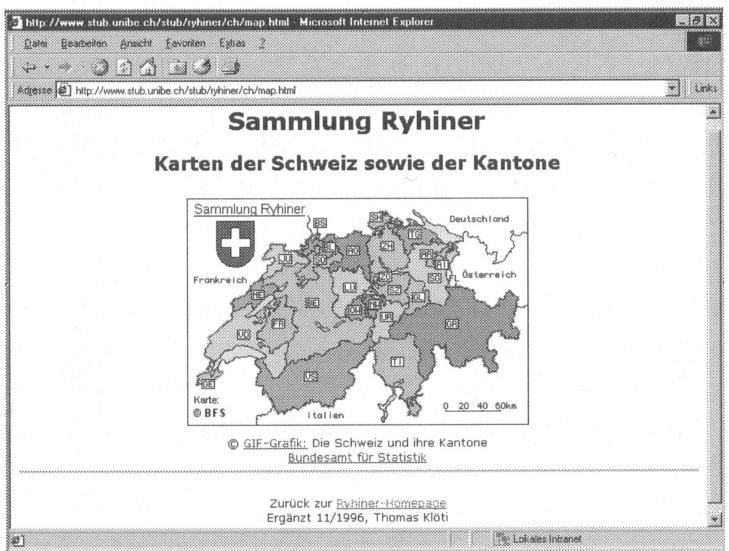

Abb. 2: Verweis-sensitive Graphik „Die Schweiz und ihre Kantone“ mit Links auf die entsprechenden HTML-Seiten 
Die Bilanz nach einem Jahr im WWW sah denn auch bereits sehr erfolgsversprechend aus. Zwischen Dezember 1995 und März 1996 wurde zum Beispiel unsere deutsch- und englischsprachige Eröffnungsseite zirka 1000 mal monatlich angeklickt, eine Zugriffsrate, die in der Folge noch weiter stark anstieg.

Zusätzlich zu diesem, damals erst via Telnet zugänglichen Bibliothekskatalog wurde für einen Teilbestand der Sammlung eine webtaugliche Datenbank verwirklicht, wobei wir dazu die Software „BestWeb Intelligent Interface Builder“ verwendeten (Abb. 3).

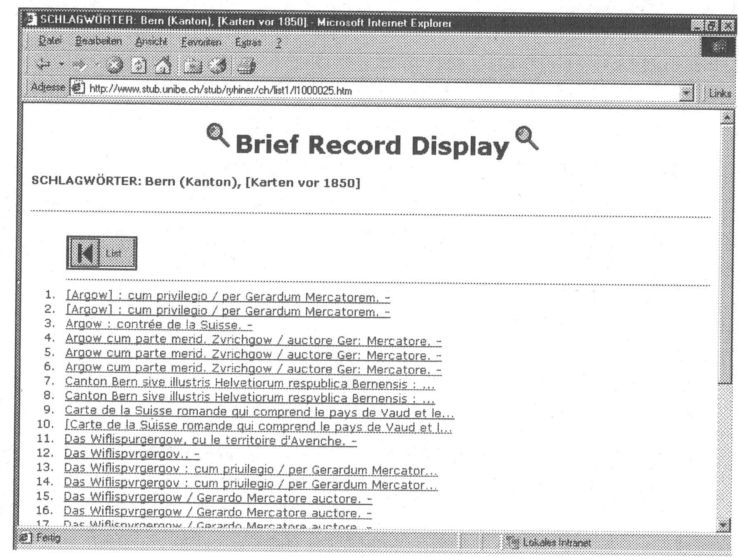

Abb. 3: Zusätzlich zum damals erst via Telnet zugänglichen Bibliothekskatalog entstand 1996 diese webtaugliche Datenbank für einen Teilbestand der Sammlung

\section{Digitales Bildarchiv}

Zur Präsentation der Sammlung im Internet gehörte bzw. gehört auch ein kleines, noch rudimentäres digitales Bildarchiv, mit dem wir Möglichkeiten aufzeigen sowie erste Erfahrungen gewinnen wollten. Dieses Angebot erwies sich bald einmal als der Spitzenreiter unseres Angebots.

Aufgrund der grossen Nachfrage erachteten wir weitere Schritte in diese Richtung als notwendig, wobei wir z. B. daran dachten, vorerst den Teilbestand mit den Schweizer Karten, Plänen und Ansichten in digitaler Form anzubieten. Da bei der Projektierung der Arbeiten eine derartige Entwicklung nicht voraussehbar war und damit auch nicht budgetiert werden konnte, standen uns für die erforderlichen Digitalisierungsarbeiten bisher keine finanziellen Mittel zur Verfügung. Daher wurde und wird auch weiterhin versucht, im Rahmen des geplanten Digitalisierungsprojekts der Stadt- und Universitätsbibliothek Bern "Berner Kultur und Geschichte im Internet", ein entsprechendes Teilprojekt in die Wege zu leiten. Die Abklärungen für 
dieses Digitalisierungsprojekt sind inzwischen so weit fortgeschritten, dass das Scannen eines Teilbestandes der Sammlung Ryhiner ab den bestehenden Mikrofilmen allmählich in greifbare Nähe rückt.

\section{Chancen und Anforderungen}

Die angestrebte Digitalisierung bietet Chancen, sie ist aber auch mit hohen Anforderungen verbunden. Die Chancen bestehen sicher darin, dass durch die Verwendung von digitalen Bildern eine Sammlung intensiv genutzt werden kann, ohne dass der wertvolle Kartenbestand Schaden leidet. Das Scannen der Mikrofilme bzw. die Digitalisierung von alten Karten ist jedoch äusserst anspruchsvoll, da auch feinste Details (z. B. Kartenschrift, Signaturen) noch lesbar sein müssen, was der Komprimierung der Dateien Grenzen setzt. Ein digitales Kartenarchiv benötigt damit sehr viel Speicherplatz und ein Benutzer muss sich, zumindest im Internet, oft mit langen Wartezeiten für die Übertragung und für den Bildaufbau abfinden. Da die Computertechnik auf eine äusserst kurze Verwendungsdauer ausgerichtet ist, sind die gemachten Vorkehrungen zudem bald einmal veraltet. Die Bestandespflege für digitale Nischenprodukte aus dem Bereich der Bibliotheken und Museen kann für eine einzelne Institution sehr aufwändig werden, da insbesondere auch ein gewichtiges Problem bei der Langzeitsicherung digitaler Informationen zu orten ist. Trotz der Faszination, die den neuen Medien zur Zeit entgegengebracht wird, muss zuletzt gesagt werden, dass das digitale Abbild zwar die in den Karten steckende Information $\mathrm{zu}$ jeder beliebigen Zeit und an jedem beliebigen Ort vermitteln kann. Das Abbild kann jedoch die Ausstrahlung, ja die Faszination, die sich bei der Beschäftigung mit originalen und damit nur sehr eingeschränkt verfügbaren Kartenblättern einstellt (z. B. anlässlich einer Ausstellung oder Spezialführung), niemals ersetzen.

Das gewählte Vorgehen, vorerst als konservierende Maßnahme eine hochauflösende Mikroverfilmung der Sammlung durchzuführen, ist aus dem Rückblick als sinnvolle Entscheidung zu betrachten. Inzwischen ist die Technik jedoch weiter fortgeschritten und die Preise für Scans sind entsprechend gesunken. Heute müsste bei einer Mikroverfilmung auch das gleichzeitige Scannen in Erwägung gezogen werden. Da auch in Zukunft weitere Innovationen zu erwarten sind, wird es - unter dem Gesichtspunkt der in der Bibliothekswelt eher knapp vorhandenen Geldmittel - sicher zweckmässig sein, vorerst einen Teilbestand in die weiterführenden Digitalisierungsvorhaben $\mathrm{ab}$ den bestehenden Mikrofilmen einzubeziehen, um damit weitere Erfahrungen gewinnen zu können. 


\section{Nachtrag (2003)}

Der Artikel vermittelt, wie der Buchtitel besagt, eine „Momentaufnahme“ des Kartenbibliothekswesens aus dem Jahre 2001. Seither gingen die Arbeiten in der Stadtund Universitätsbibliothek Bern im Hinblick auf die Sammlung Ryhiner weiter. Teile der Sammlung (Schweiz, Welt [Himmelskarten, Erdkarten], Amerika, Asien, Afrika, Australien, Biblische Geografie, Historische Karten, Titelblätter [Stand: Juni 2003]) sind bereits ab Mikrofilm durch die Firma Gubler Imaging in Märstetten gescannt worden und über den Webserver der StUB im Internet in einer Vorausschau und teilweise bereits in einer mittleren Auflösung zugänglich.

\begin{tabular}{|l|l|l|l|}
\hline Auflösung & Format & Grösse & Verwendungszweck \\
\hline 400 Pixel & JPG & $<100 \mathrm{~KB}$ & Voransicht \\
\hline 1024 Pixel & JPG & $<1 \mathrm{MB}$ & Arbeitskopie \\
\hline 4000 Pixel & JPG & Ca. 4 MB & Reproduktion \\
\hline 4000 Pixel & TIFF & Ca. $38 \mathrm{MB}$ & Masterscan \\
\hline
\end{tabular}

Zur Zeit sind die Karten des Kontinents Europa sowie Teile der Niederlande in Arbeit. Damit steht demnächst bereits ca. ein Viertel des Bestandes digital zur Verfügung. Für weitergehende Anwendungen steht auf Anfrage auch der hochaufgelöste Masterscan (der auch einen Farb- und Graukeil umfasst) zur Verfügung.

Im Februar 2003 erschien zudem zum 200. Todesjahr des Berner Staatsmannes und Kartensammlers Johann Friedrich von Ryhiner (1732-1803) der vierbändige Katalog zur Sammlung Ryhiner, der 16.528 Katalognummern und 1.786 Druckseiten umfasst. Damit liegen die Resultate der zwischen 1994 und 1998 aufgebauten Datenbank nun auch in gedruckter Form vor:

Klöti, Thomas (2003): Sammlung Ryhiner - Karten, Pläne und Ansichten aus dem 16. bis 19. Jahrhundert $=$ Ryhiner Collection - maps, plans and views from the 16th to the 19th century. 4 Bände. Bern, Stadt- und Universitätsbibliothek, 2003. 


\section{Literatur}

BARTH, Robert (1994): Von der Tontafel zur elektronischen Datenbank. (Unipress 82, 1994, S. 4-11)

GUBLER, Martin und KLÖTI, Thomas (1995): Der farbige Mikrofilm. Zwischenoriginal für konventionelle und digitale Bildarchivierungssysteme. (NIKE-Bulletin, 2/1995, S. 7-9)

HABLÜTZEL, Caroline und KLÖTI, Thomas (1995): Die Ryhiner-Sammlung und das Internet. (Stubsnase, Hauszeitschrift der Stadt- und Universitätsbibliothek Bern, 1/1995, S. 3-7)

KLÖTI, Thomas (1994): Karten in der Stadt- und Universitätsbibliothek Bern - Die Kartensammlung des Berner Staatsmannes und Geographen Johann Friedrich von Ryhiner (1732-1803). Ausstellung vom 4. Februar bis 15. März 1994. Bern, Stadtund Universitätsbibliothek, 1994. (Xerokopie.)

KLÖTI, Thomas (1995): Der virtuelle Atlas. Die Sammlung Ryhiner sowie weitere kartengeschichtlich interessante Anlegestellen im Internet. (Cartographica Helvetica 12,1995 , S. 45-47)

KLÖTI, Thomas, ÖHRLI, Markus, FELDMANN, Hans-Uli (Hrsg) (1998): Der Weltensammler. Eine aktuelle Sicht auf die 16000 Landkarten des Johann Friedrich von Ryhiner (1732-1803). (Cartographica Helvetica, Sonderheft 15, 1998) 


\section{WWW-Adressen (URLs)}

Digitale Karten - eine Herausforderung für die Kartensammlung? (H. Asche)

http://www.alexandria.ucsb.edu/ - Alexandria Project

http://libraries.mit.edu/research/ask/ask-general.html - Ask us live

http://www.ifla.org - IFLA

http://atlas.gc.ca - The national atlas of Canada online

Digitale Angebote in Kartenbibliotheken - vom Konzept zur Realisierung (J. Bühler)

http://www.maps.ethz.ch - The World of Maps - Die Welt der Karten

http://www.ethbib.ethz.ch/ks/ - Kartensammlung ETH Zürich

http://www.maps.ethz.ch/imhof.html - Virtual Library Eduard Imhof

CD-ROM-Produkte in der Kartensammlung der ETH Zürich (H. Meyer)

http://www.ethbib.ethz.ch/ks/cd-rom.html - CD-ROM-Bestand der ETH-Kartensammlung

Die Nutzung von GIS-Produkten in der Kartensammlung (J. Bühler)

http://www.kogis.ch/sikgis_d.htm - SIK-GIS-Dateninventar

Verfilmung und Digitalisierung der Sammlung Ryhiner (T. Klöti)

http://www.stub.unibe.ch/stub/ryhiner/ry-dt1.html - Ryhiner-Kartensammlung

Das Projekt "Elektronisches Kartenarchiv" an der UB Sachsen-Anhalt in Halle (J.-P. Grell) http://haweb1.bibliothek.uni-halle.de/cgi-bin/wwwlibmenu - OPAC Halle http://www.bibliothek.uni-halle.de/karten/ - Kartensammlung Halle http://www.gbv.de/ - GBV Gemeinsamer Bibliotheksverbund http://karten.bibliothek.uni-halle.de/mapwork/Sachsen-Anhalt/Eingangsbild/ - Sachsen-Anh. http://haweb1.bibliothek.uni-halle.de/cgi-bin/nphwwp3?DB=BES1.SYS8\&LANG=

DU\&EXT $=\mathrm{ON} \& \mathrm{CMD}=\mathrm{f}+$ swo + emap - Digitaler Kartenbestand

Der digitale, historische Kartenkatalog der SuUB Bremen (T. Piezunka + A. Kintrup) http://gauss.suub.uni-bremen.de - Historischer Kartenkatalog der SuUB Bremen http://www.internetvisor.de - Internet visor von megatel

Die "Virtual Library Eduard Imhof" - Kartographiegeschichte im Internet (K. Turconi) http://www.maps.ethz.ch/imhof.html - Virtual Library Eduard Imhof, deutsch http://www.maps.ethz.ch /imhof_engl.html - Virtual Library Eduard Imhof, englisch

Verweis-sensitive Indexblätter als Bestandesnachweis im Internet (W. Crom) http://www.dbiberlin.de/dbi_pub/bd_art/bd_99/99_04_07.htm

Artikel in Bibliotheksdienst 33, 1999

http://www.ethbib.ethz.ch/ks/index-bw50.html - Verweis-sensitives Indexblatt http://www.ethbib.ethz.ch/ks/tk50bw.html - Bestandesliste Baden-Württemberg 1:50.000 
Vom OPAC zum Indexblatt, vom SWB zur Online-Systematik (H. Wiesenmüller) http://www.wlb-stuttgart.de/referate/kartograph/bestand.htm - Internet-Kartenkatalog Crom http://dbl-www.sub.uni-goettingen.de/gok/ - Göttinger Online-Klassifikation

Von der verweis-sensitiven Blattübersicht zur Titelaufnahme im Bibliotheks.-Verbundkatalog (T. Klöti)

http://www.ethbib.ethz.ch/ks/indexblaetter.html - Beispiel Indexkarten http://www.swisstopo.ch/de/maps/ak/INDEX.htm - Kartensamml. Schweiz. Landestopograph. http://auriga.admin.ch:8081/ - Verbundkatalog Alexandria

\section{Kartensammlung und Internet (J. Bühler)}

http://www.ethbib.ethz.ch/ks/ - Kartensammlung ETH Zürich http://www.ethbib.ethz.ch - ETH-Bibliothek Zürich http://oddens.geog.uu.nl/index.html - Oddens: The Fascinating World of Maps http://www.maps.ethz.ch - The World of Maps - Die Welt der Karten http://opac.nebis.ch:4505/ALEPH/ - Bibliotheksverbundkatalog NEBIS http://www.ethbib.ethz.ch/ks/indexblaetter.html - Indexblätter und Bestandeslisten http://www.ethbib.ethz.ch/ks/cd-rom.html - CD-ROM-Bestand http://www.maps.ethz.ch/gdc-education.html - Bildung für Kartenkuratoren im Internet http://www.maps.ethz.ch/imhof.html - Virtual Library Eduard Imhof

\section{"Die Welt der Karten" - ein Portal zur Suche nach Karten im Internet (J. Bühler)} http://www.maps.ethz.ch - The World of Maps - Die Welt der Karten http://oddens.geog.uu.nl/index.html - Oddens: The Fascinating World of Maps http://www.lib.utexas.edu/maps/index.html - Perry-Castañeda Library Map Collection http://alexandria.sdc.ucsb.edu/other-sites/ - Alexandria Digital Library http://www.cgrer.uiowa.edu/servers/servers_references.html - Maps+References, Univ.Iowa

\section{Metadatenkatalog für Raumdaten - Beispiel Geo-Guide (M. Schüler)}

http://www.sub.uni-goettingen.de - Niedersächs.Staats- u. Universitätsbibliothek http://www.Geo-Guide.de/ - Geo-Guide http://www.MathGuide.de/ - MathGuide http://www.sub.uni-goettingen.de/vlib/ -AngloAmericanGuide http://www.virtuellefachbibliothek.de - Virtuelle Forschungsbibliothek der DFG http://www.renardus.org/ - Renardus- The clever route to information http://www.oclc.org/connexion/ - OCLC CORK: Boost your library's net value http://scout.cs.wisc.edu/research/SPT/main.html - Scout Research

\section{Wissen für Kartenkuratoren - aus dem Internet (J. Bühler)} http://www.maps.ethz.ch/gdc-education.html - LIBER Groupe des Cartothécaires - Eduction http://www.maps.ethz.ch/gdc-education2.html - Literatur zum Kartenbereich http://www.maps.ethz.ch/gdc-education4.html - GdC-Campus - Tutorials and Courses http://www.maps.ethz.ch/gdc-education6.html - Who is Who in Map Librarianship http://www.kb.nl/infolev/liber/intro.htm - LIBER Groupe des Cartothécaires 


\section{Autorenverzeichnis}

\section{Markus APPENZELLER}

Kartensammlung, ETH-Bibliothek, Rämistrasse 101, CH-8092 Zürich

Tel: +41 (1) $6322190 \quad$ E-mail: appenzeller@library.ethz.ch

Hartmut ASCHE, Prof. Dr.

Lehrstuhl Geoinformatik am Institut für Geographie, Univ. Potsdam, D-14469 Potsdam

Tel: +49 (31) $9772274 \quad$ E-mail: gislab@rz.uni-potsdam.de

Kurt BRUNNER, Prof. Dr.

Lehrstuhl für Kartographie und Topographie, Universität der Bundeswehr München,

D-85577 Neubiberg

Tel.: +49 (89) 6004-4049Ｅ-mail: kurt.brunner@unibw-muenchen.de

Jürg BÜHLER, Dr.

Kartensammlung, ETH-Bibliothek, Rämistrasse 101, CH-8092 Zürich

Tel: +41 (1) $6322184 \quad$ E-mail: juerg.buehler@library.ethz.ch

\section{Wolfgang CROM}

Kartenabteilung, Staatsbibliothek zu Berlin, Unter den Linden 8, D-10117 Berlin

Tel: +49 (30) $2661235 \quad$ E-mail: wolfgang.crom@sbb.spk-berlin.de

Jens-Peter GRELL

Kartenabteilung, Staatsbibliothek zu Berlin, Potsdamer Str. 33, D-10785 Berlin

Tel: +49 (345) $5522129 \quad$ E-mail: jens-peter.grell@sbb.spk-berlin.de

Andrea KINTRUP

Staats- und Universitätsbibliothek Bremen. Bibliothekstraße, 28359 Bremen

Tel: +49 (421) 218-2723Ｅ-mail: kintrup@suub.uni-bremen.de

Thomas KLÖTI, Dr.

Bundesamt für Landestopographie, Seftigenstrasse 264, CH-3084 Wabern/Bern

Tel: +41 (31)9632241Ｅ-mail: thomas.kloeti@lt.admin.ch

Agnes KOVACS

Inst. f. Publizistik, Wissenschaft und Medienforschung, Univ. Zürich, CH-8035 Zürich

Tel. +41 (1)634 $4663 \quad$ E-mail: agnes.kovacs@ipmz.unizh.ch

Joachim LAMATSCH

PF150, D-79001 Freiburg

Tel: +49 (761) 554409

E-mail: lamatsch.joachim@t-online.de 